\title{
Theoretical and numerical investigation of flow stability in porous materials applied as volumetric solar receivers
}

\author{
M. Becker ${ }^{\text {a }}$, Th. Fend ${ }^{\mathrm{a}, *}$, B. Hoffschmidt ${ }^{\mathrm{b}}$, R. Pitz-Paal ${ }^{\mathrm{a}}$, O. Reutter ${ }^{\mathrm{a}}$, \\ V. Stamatov ${ }^{\mathrm{c}}$, M. Steven ${ }^{\mathrm{c}}, \mathrm{D}$. Trimis ${ }^{\mathrm{c}}$ \\ a Institute of Technical Thermodynamics, German Aerospace Center, Linder Höhe, D-51147 Köln, Germany \\ ${ }^{\mathrm{b}}$ Solar-Institut Jülich, Heinrich-Mußmann-Str. 5, 52428 Jülich, Germany \\ c Institute of Fluid Mechanics, Friedrich-Alexander University Erlangen-Nuremberg, Cauerstr. 4, D-91058 Erlangen, Germany
}

Received 26 August 2005; received in revised form 30 September 2005; accepted 22 November 2005

Communicated by: Associate Editor Carlos Ramos Berumen

\begin{abstract}
This article reports results of a theoretical analysis as well as a numerical study investigating the occurrence of flow instabilities in porous materials applied as volumetric solar receivers. After a short introduction into the technology of volumetric solar receivers, which are aimed to supply heat for a steam turbine process to generate electricity, the general requirements of materials applied as solar volumetric receivers are reviewed. Finally, the main methods and results of the two studies are reported. In the theoretical analysis it is shown that heat conductivity as well as permeability properties of the porous materials have significant influence on the probability of the occurrence of flow instabilities. The numerical study has been performed to investigate the occurrence of unstable flow in heated ceramic foam materials. In the simulations a constant heat flow of radiation, that is absorbed in a defined volume, and constant permeability coefficients are assumed. Boundary conditions similar to those of the $10 \mathrm{MW}$ Solucar Solar project have been chosen. In a three dimensional, heterogeneous two phase heat transfer model it was possible to simulate local overheating of the porous structure. The parameters heat conductivity, turbulent permeability coefficient and radial dispersion coefficient have been varied systematically. Consequently, for a heat flux density of $1 \mathrm{MW} / \mathrm{m}^{2}$ a parameter chart could be generated, showing the possible occurrence of "unstable" or "stable" thermal and fluid mechanical behaviour. These numerical results are beneficial for the design of optimized materials for volumetric receivers.
\end{abstract}

(c) 2006 Elsevier Ltd. All rights reserved.

Keywords: Volumetric receiver; Flow instabilities; Solar tower technology

\footnotetext{
* Corresponding author. Tel.: +49 2203601 2101; fax: +49 220366900.
}

E-mail address: thomas.fend@dlr.de (Th. Fend).

\section{Introduction}

A lot of theoretical and experimental research work has been conducted on properties and physical restrictions of the open volumetric receiver principle (Palero et al., 2003; Garcia-Casal and Ajona, 2000; 


\section{Nomenclature}

$p \quad$ pressure

$\mu_{\text {DYN }}$ dynamical viscosity

$K_{1} \quad$ viscous permeability coefficient

$K_{2} \quad$ inertial permeability coefficient

$\rho_{\mathrm{F}} \quad$ density of the fluid

$U_{0} \quad$ velocity of the fluid

$R \quad$ specific gas constant $(=287 \mathrm{~J} /(\mathrm{kg} \mathrm{K}))$

$T$ temperature

$T_{0} \quad$ inlet temperature
$T_{\text {out }} \quad$ outlet temperature

$\dot{m} \quad$ mass flow density

$I_{0} \quad$ solar radiation flux

$C_{P \mathrm{~F}} \quad$ specific heat of fluid (air)

$\beta \quad$ correction factor

$\sigma \quad$ Stefan-Boltzmann constant $(=5.6696 \times$ $10^{-8} \mathrm{~W} \mathrm{~m}^{-2} \mathrm{~K}^{-4}$ )

$v \quad$ velocity vector $\left(=v_{1}, v_{2}, v_{3}\right)$ in numerical calculations
Hoffschmidt, 1997). In several independent experiments from the field of solar applications concerning the flow through porous materials there have been indications showing a relationship between solar flux and flow speed (Buck et al., 1993; Buck, 2000; Muir et al., 1994). Local high solar flux leads to a low mass flow and high material temperatures. Local low solar flux leads to a high mass flow with a low material temperature (Fig. 1). This means that the absorber material can exceed the application temperature locally which leads to its destruction although the average air outlet temperature is low. The main cause of this behaviour is the temperature dependent increase of the viscosity of air. A similar effect of instability can be observed for example at the degassing of polymers, where a highly inhomogeneous mass flow distribution is caused by a set of openings for the degassing process (Ianniruberto et al., 1992). Because in this case the viscosity decreases with rising temperature, this phenomenon only occurs, when the material temperature is lower than the fluid temperature (cooling).

Several theoretical approaches (Kribus et al., 1996) or numerical simulations (Hoffschmidt, 1997; Buck, 2000; Pitz-Paal et al., 1997) lead to a

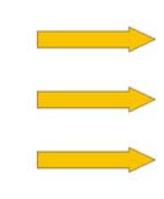

solar radiation
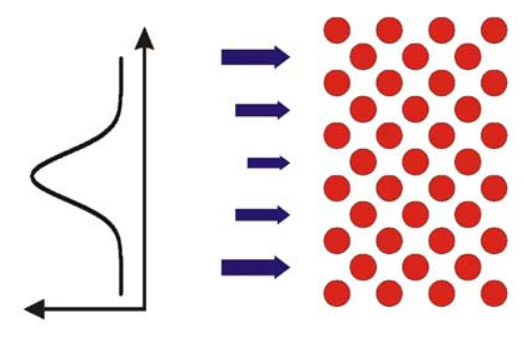

air flow cellular material
Fig. 1. Instabilities in the flow through porous materials used in solar applications. fairly good agreement between the calculations and the experiments and general tendencies could be shown. The most important influence on flow stability comes from the pressure loss characteristic of the porous media. With a linear dependency of the pressure loss on the flow velocity (Darcy flow) instabilities can occur, with a pure quadratic dependency (Dupuit, Forchheimer) these do not occur. The problem is, that in solar applications usually there is a mixture of both linear and quadratic behaviour. In the following it will be shown with a simple model under which condition instabilities can occur in the flow through porous media.

\section{Simple theoretical model}

In the flow through a porous structure the mass flow density is determined by the pressure difference between the two sides of the structure. The pressure drop is produced by a blower. It is the same across the whole structure, because there is an instant pressure equalization on both sides. Instability occurs, when one certain pressure drop can cause different mass flow densities to flow.

The pressure drop across the sample can be described by Darcy's law in the Forchheimer extension:

$-\frac{\mathrm{d} p}{\mathrm{~d} x}=\frac{\mu_{\mathrm{DYN}}}{K_{1}} U_{0}+\frac{\rho_{\mathrm{F}}}{K_{2}} U_{0}^{2}$.

where $p$ denotes the pressure, $x$ the coordinate in the direction of the flow, $K_{1}$ and $K_{2}$ the viscosity and the inertial coefficient, $\mu_{\mathrm{DYN}}$ the dynamical viscosity, $\rho$ the density of the fluid and $U_{0}$ the velocity of the fluid.

If air is used as a fluid, the ideal-gas law can be taken as valid in a wide temperature range: 
$p=\rho R T$,

with the specific gas constant $R, 287 \mathrm{~J} /(\mathrm{kg} \mathrm{K})$, and the air temperature $T$. The dependence of the dynamic viscosity on the temperature of the air can be approximated by

$\mu_{\mathrm{DYN}}(T)=\mu_{\mathrm{DYN}}\left(T_{0}\right)\left(\frac{T}{T_{0}}\right)^{0.7}$.

Further on the mass flow density can be expressed as

$\dot{m}=\rho U_{0}$.

Using Eqs. (2)-(4) in Eq. (1) gives:

$-p d p=\left(\frac{\mu_{\mathrm{DYN}}\left(T_{0}\right)}{K_{1} T_{0}^{0.7}} T^{0.7} \dot{m}_{\mathrm{A}} R T+\frac{R T \dot{m}_{\mathrm{A}}^{2}}{K_{2}}\right) \mathrm{d} x$.

This equation can be integrated assuming that one is looking at a long absorber and that the temperature distribution along the length of the absorber can be well approximated by the outlet temperature. In practice the temperature rise of the fluid from inlet to outlet takes place in the first few millimetres, whereas absorber sample thicknesses range in the order of several centimetres due to stability reasons. This integration gives:

$\frac{p_{0}^{2}-p_{\text {out }}^{2}}{2}=\left(R \frac{\mu_{\mathrm{DYN}}\left(T_{0}\right)}{K_{1} T_{0}^{0.7}} T_{\text {out }}^{1.7} \dot{m}+\frac{R T_{\text {out }} \dot{m}^{2}}{K_{2}}\right) L$.

Here $L$ denotes the length of the absorber, $T_{0}$ and $T_{\text {out }}$ are respectively inlet and outlet temperature of the air . Now considering an absorber and its energy balance neglecting radial heat transfer

$I_{0}=\dot{m} C_{P \mathrm{~F}}\left(T_{\text {out }}-T_{0}\right)+\beta \sigma T_{\text {out }}^{4}$

with the solar radiation flux $I_{0}$, which is shining on the surface of the absorber, $C_{P \mathrm{~F}}$ the specific heat of the air, $\sigma$ is the Stefan-Boltzmann-constant and $\beta$ is a correction factor, which describes the thermal losses through radiation. If $\beta$ is smaller than 1 , then the surface temperature of the outside of the absorber is smaller than the air outlet temperature ("volumetric operation') and the radiation losses are smaller. This is the case, when the solar radiation can get into the inside of the porous structure and the incoming air cools the front surface well. If $\beta>1$ the conditions are similar to those in a pipe receiver. The surface temperature is greater than the air outlet temperature (no 'volumetric operation').

Rearranging the energy balance for the mass flow density and putting it into the integrated pressure drop equation gives:

$$
\begin{aligned}
\frac{p_{0}^{2}-p_{\text {out }}^{2}}{2}= & R L \frac{\mu_{\mathrm{DYN}}\left(T_{0}\right)}{K_{1} T_{0}^{0.7}} T_{\text {out }}^{1.7} \frac{I_{0}-\beta \sigma T_{\text {out }}^{4}}{C_{P \mathrm{~F}}\left(T_{\text {out }}-T_{0}\right)} \\
& +\frac{R L T_{\text {out }}}{K_{2}}\left(\frac{I_{0}-\beta \sigma T_{\text {out }}^{4}}{C_{P \mathrm{~F}}\left(T_{\text {out }}-T_{0}\right)}\right)^{2} .
\end{aligned}
$$

In the case of the structure having a linear pressure drop relation, that is $K_{2}=\infty$, the equation above becomes a lot simpler. The graph of the quadratic pressure difference versus the temperature shows what happens in the case of instability. If there are one or more temperatures and therewith connected mass flows possible to the same pressure drop then there is instability. In the graph lines with constant pressure drop are drawn, which intersect the curves with high solar flux in three points. So to the same pressure drop different temperatures are possible. Parts of the absorber can have a low mass flow through them and other parts a high mass flow. The low mass flow can lead to local overheating and thus to the destruction of the absorber.

Fig. 2 shows the quadratic pressure drop as a function of the temperature for different solar fluxes and a material with purely linear pressure drop characteristic $\left(K_{2}=\infty\right)$. One can see, that instability only exists above a certain solar flux.

Looking at instability mathematically an ambiguity can only occur, if the curve of the pressure drop shows a zero-point in the derivate by the outlet temperature which lays in the physically relevant range between the maximum of the inlet temperature $T_{0}$ and the maximum temperature $T_{\max } . T_{\max }$ is the temperature which is reached when no mass flow is flowing:

$T_{\max }=\sqrt[4]{\frac{I_{0}}{\sigma}}$

This zero-point of the derivative of the pressure difference depends solely on $T_{\text {out }}, T_{0}, \beta, \sigma$ and $I_{0}$. Set-

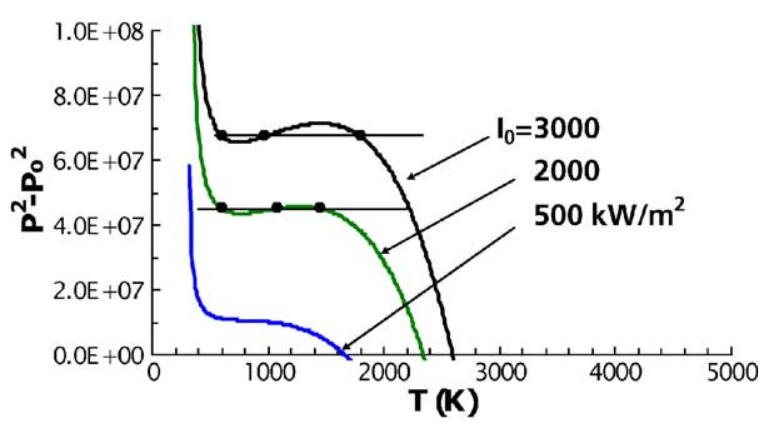

Fig. 2. Quadratic pressure drop versus the air temperature for different solar fluxes. 
ting the derivative to zero leads to the following equation:

$0=0.7 I_{0} T_{\text {out }}+5.7 \beta \sigma T_{0} T_{\text {out }}^{4}-4.7 \beta \sigma T_{\text {out }}^{5}-1.7 T_{0} I_{0}$.

This equation can only be satisfied, if the solar flux is above a critical value, below this critical value there is no solution. This value can be obtained by solving this equation for $I_{0}$ and finding the minimum of the obtained expression. This minimum is found at $T_{\text {out }}=2.95 T_{0}$. This gives a critical flux above which instabilities can occur:

$I_{0, \text { crit }}=1694 \beta \sigma T_{0}^{4}$.

For example for $\beta=1$ and $T_{0}=300 \mathrm{~K}$ this means a critical flux of $778 \mathrm{~kW} / \mathrm{m}^{2}$.

The above calculation was only valid for a porous media with a purely linear pressure drop characteristic. Now looking at the curves of the quadratic pressure drop difference for the case of $K_{2} \neq \infty$, the tendency for the case of constant solar flux can be seen in Fig. 3. A change in the pressure drop characteristic of the absorber has a significant influence on the curves. The lower the values of $K_{2}$ the less probable instabilities become. The values of $K_{1}$ and $K_{2}$ for the curves shown in the graph are of a realistic magnitude. For example the SiC parallel channel catalyst carrier with a cell density of about 80 CPSI has $K_{1}=10^{-7} \mathrm{~m}^{2}$ and $K_{2}=0.011 \mathrm{~m}$. For foam ceramics the values of $K_{2}$ are even less and can be smaller than $10^{-4} \mathrm{~m}$. This means practically that when using a ceramic foam as a volumetric receiver at a solar flux of 2000 sun instabilities are not expected.

The mathematical description of the more general case is a little more complicated but can be con-

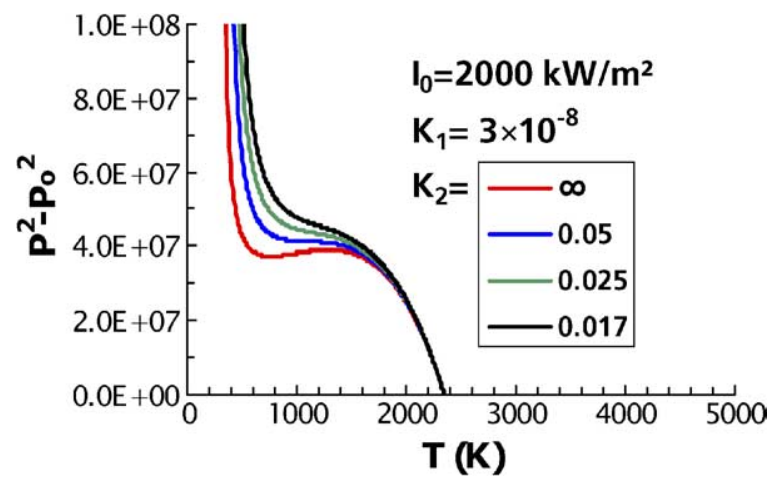

Fig. 3. The quadratic pressure drop difference as a function of the air outlet temperature for several values of the inertial coefficient. ducted in a similar way. Calculations give a critical ratio of $K_{1}$ and $K_{2}$ at:

$\frac{K_{1}}{K_{2}}=1.94 \times 10^{-6} \mathrm{~m}$

above which instabilities are not to be expected.

In the above discussed simple model used to study the systematic dependency on the parameters under which instabilities occur not all parameters are included. A further important parameter of flow stability is the thermal conduction of the absorber. It allows a heat flow in the case of an inhomogeneous temperature distribution and thus can level out local hot regions. The better the heat conduction in the absorber is, the less possible instabilities get.

\section{Numerical simulations}

The aim of the numerical simulations was to investigate the flow behaviour of the porous bodies under high temperature conditions. Especially the conditions under which flow and thermal instabilities occur. For these simulations a large amount of experimental data of the porous media, e.g. the effective heat conductivity, absorptivity or the volumetric heat transfer coefficient is necessary. A short review of the experimental set-ups to determine these quantities was written by Fend et al. (2004). By varying the properties of the materials in the simulations their tendency to the occurrence of instabilities was characterized. In the simulations the finite volume method was used in combination with a heterogeneous heat transfer model for the porous body.

In the following the basic equations (transport of mass, momentum and energy) in tensor notation for the calculation of a steady state flow with heat exchange but without reactions are written. To account for the porous medium these equations have to be adapted to the porous medium.

$$
\begin{aligned}
& \frac{\partial\left(\rho v_{j}\right)}{\partial x_{j}}=0, \\
& \frac{\partial}{\partial x_{j}}\left(\rho v_{j} v_{i}-\mu \frac{\partial v_{i}}{\partial x_{j}}\right)=-\frac{\partial P}{\partial x_{i}}+\frac{\partial}{\partial x_{j}}\left(\mu \frac{\partial v_{j}}{\partial x_{i}}\right)+\rho g_{i} \\
& \frac{\partial}{\partial x_{j}}\left(\rho v_{j} C_{P} T_{\mathrm{g}}-\lambda \frac{\partial T_{\mathrm{g}}}{\partial x_{j}}\right)=\frac{\partial}{\partial x_{j}}\left(\sum_{k=1}^{N_{\mathrm{S}}} \rho h_{k} D_{k} \frac{\partial Y_{k}}{\partial x_{j}}\right)
\end{aligned}
$$

where $v=\left(v_{1}, v_{2}, v_{3}\right)$ is the velocity vector with respect to the Cartesian coordinates $\left(x_{1}, x_{2}, x_{3}\right)$. The density $\rho$ was calculated for a mixture of perfect 
gases using the ideal-gas law. The other properties of the gas (dynamic viscosity, heat conductivity) are calculated as a mixture value of the properties of the pure gases by using semi-empirical correlations (Wilke, 1950; Bird et al., 1960; Mathur et al., 1967). The properties of the pure species are estimated with the help of the kinetic gas theory (Hirschfelder et al., 1954; Warnatz, 1982). Information about the heat capacity as well as further thermodynamic data can be found in the CHEMKIN-II database (Kee et al., 1992).

\subsection{Modelling of flow and heat transfer effects in porous media}

In heat and mass transfer through porous media the two phases solid and fluid are present. It is very difficult to account for the detailed resolved geometry of the porous structure. A common approach to overcome this difficulty is to average over the volume of the structure. In this paper a heterogeneous model is considered that treats the solid and gas phase separately, but the phases are coupled via a convective heat exchange term.

When a porous medium is introduced into a pipe it causes some additional pressure drop. This pressure drop depends on the properties of the porous medium. Second order polynomials are used to describe this pressure drop according to the Forchheimer equation for the $i$-direction.

$\frac{\mathrm{d} p}{\mathrm{~d} x_{i}}=-\frac{\mu}{k_{1, i j}} v_{\mathrm{s}, j}-\frac{\rho}{k_{2, i j}}\left|v_{\mathrm{s}, j}\right| v_{\mathrm{s}, j}$,

where $v_{s, j}$ is the superficial velocity based on the sectional area of the empty tube. The tensors $k_{1, i j}$ and $k_{2, i j}$ are the linear and turbulent permeability coefficients that describe the pressure loss in the porous medium. These coefficients can be determined by simple experiments. The mass conservation equation for the porous medium then reads:

$\frac{\partial\left(\rho v_{s, j}\right)}{\partial x_{j}}=0$.

For the separate treatment of the two phases the following energy equations are used:

Gas phase:

$$
\begin{aligned}
& \frac{\partial}{\partial x_{j}}\left(\rho v_{\mathrm{s}, j} C_{P} T_{\mathrm{g}}-\lambda \varepsilon \frac{\partial T_{\mathrm{g}}}{\partial x_{j}}\right) \\
& =\frac{\partial}{\partial x_{j}}\left(\sum_{k=1}^{N_{\mathrm{s}}} \rho h_{k} \varepsilon D_{k} \frac{\partial Y_{k}}{\partial x_{j}}\right)+\alpha A_{\mathrm{V}}\left(T_{\mathrm{s}}-T_{\mathrm{g}}\right) .
\end{aligned}
$$

Solid phase:

$\frac{\partial}{\partial x_{j}}\left(-\lambda_{\text {eff }}(1-\varepsilon) \frac{\partial T_{\mathrm{s}}}{\partial x_{j}}\right)=\alpha A_{\mathrm{V}}\left(T_{\mathrm{s}}-T_{\mathrm{g}}\right)$,

where $\varepsilon$ is the porosity of the structure, $\alpha$ is the heat transfer coefficient, $A_{\mathrm{V}}$ is the specific surface, $T_{\mathrm{s}}$ is the solid temperature and $\lambda_{\text {eff }}$ is the effective heat conductivity.

$\lambda_{\mathrm{eff}, i}=\lambda_{\mathrm{eff}, 0}+\frac{\rho \cdot U_{0} \cdot c_{p, \mathrm{f}} \cdot d}{K_{i}}, \quad i=a x, \mathrm{rad}$.

The effective heat conductivity is borrowed from the modelling of the heat conductivity in fixed beds. It consists of the heat conductivity without flow $\left(\lambda_{\text {eff, },}\right)$, that incorporates the effects of conduction and radiation, and the additional heat conductivity by dispersion within the porous medium. All of the described equations are solved by using the finite volume method.

\subsection{Boundary conditions and procedure for the calculation of hot spots}

For the simulations a simple Cartesian grid consisting of 64000 hexahedron elements that was separated into three blocks was used (Fig. 4). The left block represents the boundary conditions of the inlet flow into the porous medium. The middle block represents the porous medium and the righthand block the outflow of the hot air. Boundary condition values and properties of the porous medium used are listed in Table 1. In the direction perpendicular to the flow, the boundary conditions were set to zero gradients for all calculated variables.

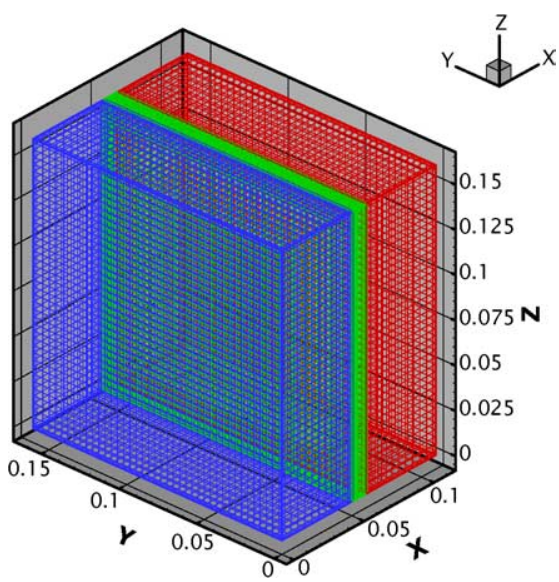

Fig. 4. Grid for the hot spot simulations. 
Table 1

Boundary condition values and properties of the porous medium

\begin{tabular}{ll}
\hline Variable & Used values \\
\hline Inlet velocity $[\mathrm{m} / \mathrm{s}]$ & 1 \\
Inlet temperature $[\mathrm{K}]$ & 300 \\
Extinction coefficient $\left[\mathrm{m}^{-1}\right]$ & 400 \\
Volumetric heat transfer & $1 \times 10^{5}$ \\
$\quad$ coefficient $\left[\mathrm{W} \mathrm{m}{ }^{-3} \mathrm{~K}^{-1}\right]$ & 1 \\
Heat flux density $\left[\mathrm{MW} / \mathrm{m}^{2}\right]$ & $10 / 5 / 1 / 0.5 / 0.1$ \\
$K_{\text {rad }}$ & $9.3 \times 10^{-10}$ \\
$K_{1}\left[\mathrm{~m}^{2}\right]$ & $1 \times 10^{20} / 1 \times 10^{-3} / 1 \times 10^{-4}$ \\
$K_{2}[\mathrm{~m}]$ & $0.01 / 0.1 / 1 / 10$ \\
$\lambda_{\text {eff, } 0}[\mathrm{~W} / \mathrm{m} / \mathrm{K}]$ &
\end{tabular}

At first the flow and temperature field was solved for homogeneous boundary conditions and a homogeneous incident radiative heat flux density of $1 \mathrm{MW} / \mathrm{m}^{2}$. After that the calculations were continued from the previous run and a small area with a much higher incident radiative heat flux density was introduced to get start field with a hot spot. When a hot spot was created this high heat flux density was switched off again and the calculations were continued with homogenous heat flux density. Depending on the properties of the porous medium this local temperature peak spread and maintained or it vanished (see Fig. 5, $y-z$-plane at $x=2 \mathrm{~mm}$ ).

The same procedure was repeated but with a change in the size of the introduced hot spot to assure that the final solution is independent of the artificially introduced error. When performing this calculation with an initial hot spot that consists of an area four times higher than in the first run, the size of the hot spot in the final solution was the same. By this the independence on the initial error could be shown.

After that 60 computations with different properties of the porous medium were performed. For the properties of the porous medium and the boundary conditions the following values were used and varied systematically in the simulations. In Fig. 6 an example of 12 calculations is shown.

From the "hot spot matrix" of Fig. 6 the dependency of the size of the obtained hot spot on the properties of the porous medium can be seen. At higher values of the effective heat conductivity is size of the hot spot smaller. The same dependency can be observed when looking at the value of the turbulent permeability coefficient. As already shown in the theoretical analysis the probability for the occurrence of a hot spot is higher when the pressure drop of the porous medium is mainly a linear function. The higher the turbulence effects get influence the smaller is the hot spot.

\section{Conclusions}

Through theoretical considerations and numerical simulations it could be shown, that there are certain material and boundary condition parameters, which can lead to flow instabilities. Fortunately there is also a large region of material parameters which lead to a save operation of a volumetric receiver. Considering the given boundary conditions of a certain set-up it is possible to find suitable material parameters which allow a save operation of the solar receiver. By using suitable receivers with a high heat conductivity and a quadratic behaviour of the pressure drop correlation, a high ratio of $K_{1}$ to $K_{2}$, like ceramic foams, instabilities can be avoided. If one is able to measure heat conductivity, permeability, heat transfer and radial dispersion of a certain sample, a simulation instead of an experiment in concentrated solar radiation can be conducted, to qualify the sample as a possible absorber material.
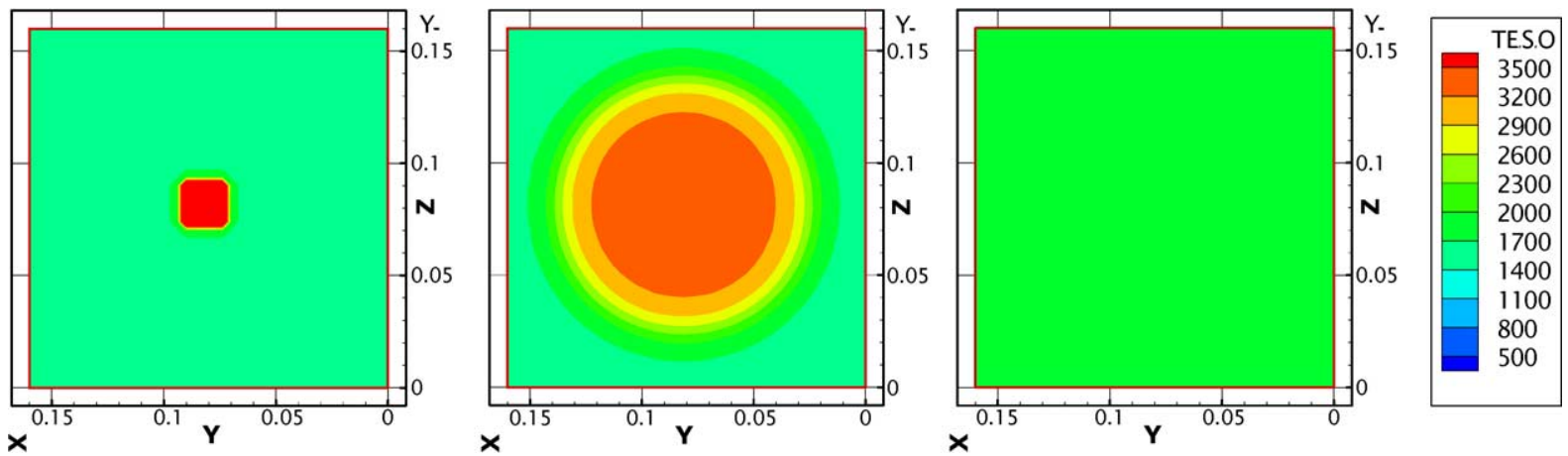

Fig. 5. Introduced hot spot/hot spot maintained at final result/hot spot vanished at final result. 

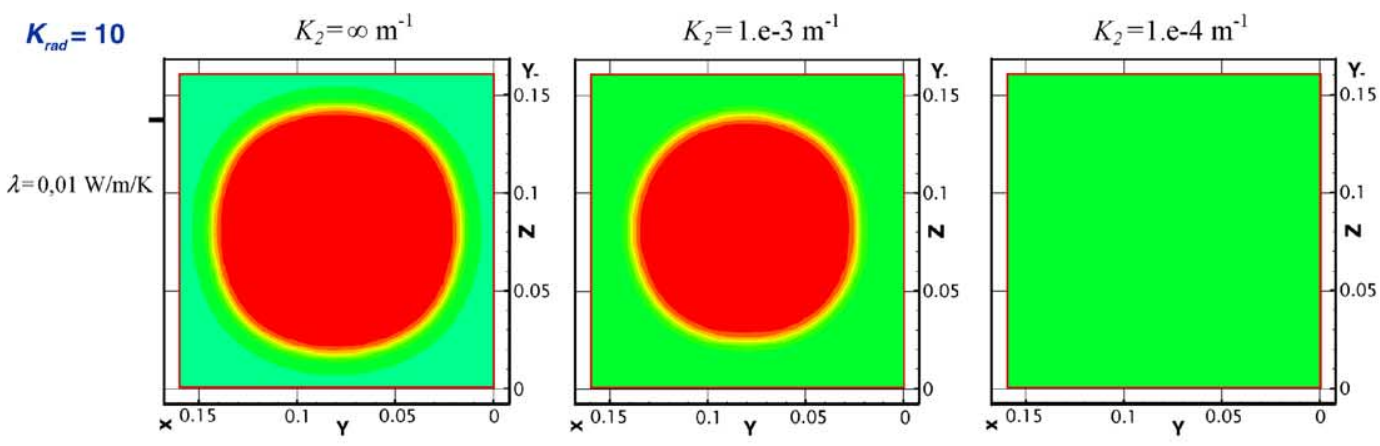

vertical slice of the $\mathrm{y}$-z-plane, at $\mathrm{x}=2 \mathrm{~mm}$
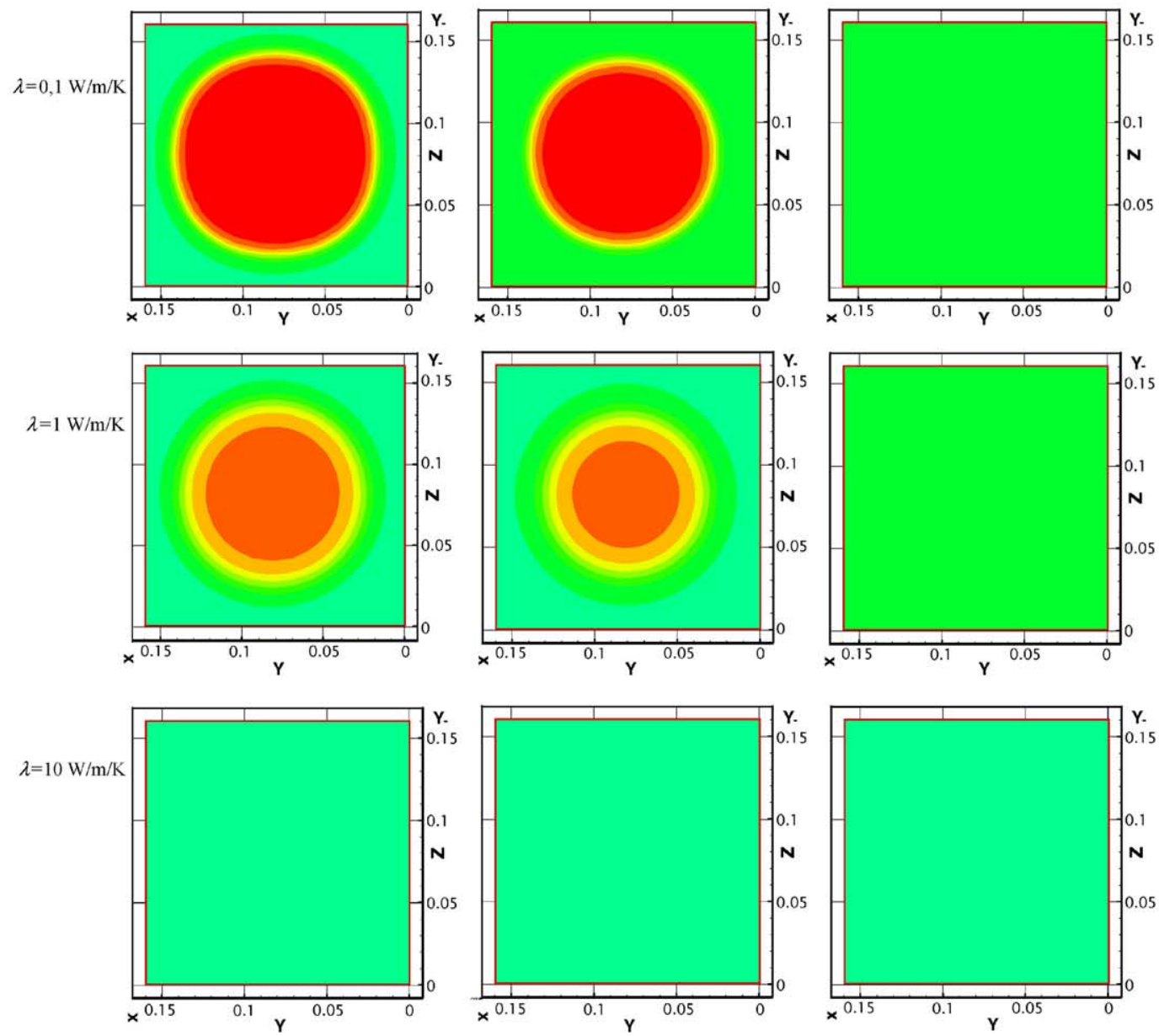

Fig. 6. Obtained results for 12 combinations of properties of the porous medium.

\section{Acknowledgement}

This study is based on the project SOLPOR, which was funded by the German Ministry of Education and Science. The help of the ministry is gratefully acknowledged.

\section{References}

Bird, R.B., Stewart, W.E., Lightfood, E.N., 1960. Transport Phenomena. John Wiley \& Sons, New York.

Buck, R. 2000. Massenstrom-Instabilitäten bei volumetrischen Receiver-Reaktoren, Fortschrittsberichte VDI Reihe $3 \mathrm{Nr}$. 648, VDI Verlag, Düsseldorf. 
Buck, R., Biehler, T., Heller, P. 1993. Advanced volumetric receiver-reactor for solar methane reforming. In: Sánchez, M., Macias, M. (Eds.), Proceedings of the 6th IEA-Symposium on Solar Thermal Concentrating Technologies, vol. I, pp. 395405.

Fend, Th., Hoffschmidt, B., Pitz-Paal, R., Reutter, O., Rietbrock, P., 2004. Porous materials as open volumetric solar receivers: experimental determination of thermophysical and heat transfer properties. Energy 29 (5-6), 823-833.

Garcia-Casal, X., Ajona, J.I., 2000. The duct selective volumetric receiver: potential for different selectivity strategies and stability issues. Solar Energy 67 (4-6), 265-268.

Hirschfelder, J.O., Curtiss, C.F., Bird, R.B., 1954. Molecular Theory of Gases and Liquids. John Wiley \& Sons, New York.

Hoffschmidt, B. 1997. Vergleichende Bewertung verschiedener Konzepte volumetrischer Strahlungsempfänger. Deutsches Zentrum für Luft- und Raumfahrt. ISRN DLR-FB 97-35. ISSN 1434-8454.

Ianniruberto, G., Maffetone, P.L., Astarita, G., 1992. Stability of multislit devolatilization of polymers. AIChE Journal 39, 140-148.

Kee, R.J., Rupley, F.M., Miller, J.A. 1992. The Chemkin Thermodynamic Data Base. Sandia National Laboratories Report SAND-8215B.

Kribus, A., Ries, H., Spirkl, W., 1996. Inherent limitations of volumetric solar receivers. Journal of Solar Energy Engineering $118,151-155$.
Mathur, S., Tondon, P.K., Saxena, S.C., 1967. Thermal conductivity of binary, ternary and quaternary mixture of rare gases. Molecular Physics 12, 569-573.

Muir, J.F., Hogan, R.E., Skocypec, R.D., Buck, R., 1994. Solar reforming of methane in a direct absorption catalytic reactor on a parabolic dish, I - Test and analysis. Solar Energy 52 (6), 467-477.

Palero, S., Romero, M., Estrada, C.A. 2003. Experimental investigation in small scale volumetric solar receivers to study the mass flow instability and its comparison to theoretical models. In: Proceedings of the ISES Solar World Congress 2003 "Solar Energy for a Sustainable Future", June 14-19, 2003, Göteborg, Sweden.

Pitz-Paal, R., Hoffschmidt, B., Böhmer, M., Becker, M., 1997. Experimental and numerical evaluation of the performance and flow stability of different types of open volumetric absorbers under non-homogeneous Irradiation. Solar Energy 60 (3-4), 135-150.

Warnatz, J., 1982. Influence of transport models and boundary conditions on flame structure. In: Peters, N., Warnatz, J. (Eds.), Numerical Methods in Flame Propagation. Vieweg, Wiesbaden.

Wilke, C.H., 1950. A viscosity equation for gas mixtures. Journal of Chemical Physics 18, 517-518. 